\title{
Sample size: how many participants are needed in a cohort study?
}

This Endgames article (BMJ 2014;349:g6557, doi:10.1136/bmj. g6557) contains an error in the last sentence of the third paragraph of the answers section. This sentence should have read: "An improvement in precision requires an increased sample size (b is true); for example, to have precision within three percentage points for the estimated prevalence of $20 \%$ would require a sample size of 683 [not 1537, as was published] participants."

Cite this as: BMJ 2014;349:g6806

(๑) BMJ Publishing Group Ltd 2014 